\title{
Interesses e Perceções de Competência na Infância: Um Estudo Exploratório no Contexto Português
}

\author{
Interests and Competence Perceptions in Childhood: An Exploratory Study in the \\ Portuguese Context
}

\author{
Maria Odília Teixeira ${ }^{1}$ e Márcia Laranjeira ${ }^{2}$
}

\begin{abstract}
Resumo
O estudo analisa as propriedades psicométricas da versão Portuguesa do Inventário de Atividades para Crianças - Revisto (ICA-R), numa amostra de crianças $(\mathrm{N}=228)$ do $4^{\circ}$ ano de escolaridade. Os dados são examinados no modelo de Holland, de acordo com premissas desenvolvimentistas e sociocognitivas que salientam fatores sociais e escolares na formação do autoconceito. A análise em componentes principais revela uma estrutura dos interesses e das crenças de capacidade consistente com o modelo de Holland e com atribuições de género e preferências escolares. O conjunto dos dados indica que a versão portuguesa do ICA$\mathrm{R}$ é metrologicamente precisa e válida, e aponta dados positivos para a validade intercultural da medida e do modelo de Holland. As potencialidades do ICA-R são discutidas na investigação sobre o processo de formação do autoconceito e na intervenção educacional de carreira.
\end{abstract}

Palavras-chave: interesses, competências, autoconceito, género, experiências escolares

\begin{abstract}
This study analyzes the psychometric properties of the Inventory of Children's Activities - Revised (ICA-R), in a sample of 4th grade children $(\mathrm{N}=228)$. Data are examined base upon Holland's Personality Type Model, according to developmental and socio-cognitive assumptions that emphasize social and academic factors in the formation of self-concept. Principal component analysis reveals a structure of interests and competence perceptions consistent with the Holland's model, as well as with gender attributions and school preferences. The data set indicates that the Portuguese version of ICA-R is metrologically accurate and valid, and points out the intercultural validity of the measure and of Holland's model. The potential of the ICA-R is discussed in the context of research on the process of self-concept formation and career educational intervention.
\end{abstract}

Keywords: interests; competences, self-concept, gender, school experiences

\footnotetext{
${ }^{1}$ Doutoramento. Faculdade de Psicologia da Universidade de Lisboa. Alameda da Universidade, 1649-013, Lisboa, Portugal. Tel.: +351917872583. E-mail: moteixeira@psicologia.ulisboa.pt

${ }^{2}$ Mestrado. Colégio do Amor de Deus, Educational Psychologist. Avenida de Sintra 1451, 2756-502 Alcabideche, Cascais, Portugal. Tel.: +351965731585. E-mail: marcia.laranjeira@campus.ul.pt
} 


\section{Introdução}

No domínio vocacional, o autoconceito representa um construto multidimensional, em que interesses, valores e competências formam a base de construção e sustentação da carreira (Super, 1990). A formação do autoconceito tem génese na infância e inscreve-se em processos de aprendizagem, que envolvem fatores de natureza pessoal, social, cultural, familiar e escolar (e.g. Ahn, Usher, Butz, \& Song, 2016; Brown \& Lent, 2017; Bussey \& Bandura, 1999; Flores, Navarro, \& Rasheed, 2017; Fouad \& Santana, 2017; Hendricks, 2016; Høigaard, Kovac, Øverby, \& Haugen, 2015). No desenvolvimento vocacional, a infância é reconhecida como período crítico das aquisições que estão na base dos interesses, valores e atitudes (Seligman, 1994) e da identidade vocacional na idade adulta (Herr \& Cramer, 1996).

O autoconceito é definido como construto hipotético, que incorpora componentes cognitivas, afetivas e emocionais (Pinxten, Marsh, Fraine, Van Den Noortgate, \& Van Damme, 2014), e de forma simples é entendido pela perceção que o indivíduo tem de si próprio (Vaz Serra, 1988), nos diversos espaços e domínios comportamentais (e.g. social, académico ou vocacional).

\section{Formação do Autoconceito Vocacional: Interesses e Competências}

A Teoria da Circunscrição e Compromisso de Gottfredson (1981, 1996, 2002) explica a formação do autoconceito vocacional com base nas dimensões género e prestígio. Neste quadro teórico, a formação do autoconceito é entendida como um processo de eliminação progressiva das alternativas vocacionais não congruentes com autorrepresentações de género e classe social. As crianças entre os 7 e os 9 anos estabelecem um mapa cognitivo de profissões baseado nos estereótipos de género; as meninas tendem a representar atividades associadas ao universo feminino (e.g. cabeleireira, professora), e os rapazes preferências profissionais atribuídas à masculinidade (e.g. futebolista, engenheiro) (Iskander, Gore, Furse, \& Bergenson, 2013; Laranjeira, 2015). Estas diferenças emergem também no contexto escolar e nas próprias brincadeiras das crianças, com as meninas a privilegiarem língua portuguesa e a brincarem em díade com a melhor amiga, e os rapazes a preferirem matemática e jogos de força física realizados em grupo (Laranjeira, 2015; Teixeira, 2014). Mais tarde, por volta dos 10 anos, as crianças circunscrevem as preferências numa hierarquia social de prestígio (e.g. médico, jornalista, advogado), ressurgindo também o fator género, sobretudo nos rapazes (Teixeira, 2009). Numa fase posterior, em plena adolescência, entre os 14 e os 16 anos, emergem os fatores psicológicos (Gottfredson, 1996, 2002), considerados normalmente como determinantes pessoais da carreira (interesses, valores, aptidões, competências) (e.g. Super, 1990). Estas dimensões psicológicas surgem no âmbito do processo de restrições sucessivas do desenvolvimento do autoconceito, em que variáveis sociais podem comprometer o pleno desenvolvimento dos domínios cognitivos e vocacionais. Em contexto social desfavorecido permanece o fator género nas aspirações das crianças, mas as preferências profissionais estabelecem-se num espaço social mais restrito e de baixo prestígio como vender nas feiras ou trabalhar na construção civil, respetivamente nas crianças de etnia cigana e de origem africana (Teixeira, 2014). Neste âmbito, muitos trabalhos suportam a influência de variáveis sociais e culturais (e.g. expectativas, nível socioeconómico, género, etnia) nos interesses e nas aspirações das crianças (e.g. Bandura, Barbaranelli, Caprara, \& Pastorelli, 2001; Bussey \& Bandura, 1999; Flores et al., 2017; Fouad \& Santana, 2017; Laranjeira \& Teixeira, no prelo). Estas variáveis sociais influenciam o autoconceito, mas também a motivação, a aprendizagem e as escolhas que firmam a construção da carreira, ao longo da vida. De acordo com Gottfredson (1996, 2002), as crianças recriam as condições de desigualdade social do seu próprio meio, ainda antes de poderem experimentar as dificuldades e obstáculos realmente impostos. A associação entre género e interesses é produto de estereótipos socialmente enraizados e perpetuados pelas práticas educativas, nomeadamente pais e professores que frequentemente transmitem expectativas e exigências diferenciadas para rapazes e raparigas (Lazarides \& Watt, 2015; 
Gunderson, Ramirez, Levine, \& Beilock, 2012; Silva, 2016).

Numa visão complementar e integradora, a Teoria Sociocognitiva da Carreira (TSSC) (Lent et al., 1994; Lent \& Brown, 2017; Brown \& Lent, 2017) explica a formação do autoconceito através dos processos de aprendizagem, na aceção de conceber o funcionamento psicológico na relação triádica entre pessoa, situação e comportamento (Bandura, 1977). Os interesses são desenvolvidos a partir das crenças de autoeficácia, que, por sua vez, são organizadas nas aprendizagens do dia-adia da criança (Lent et al., 1994).

Na teoria de Bandura (1997) são fontes de autoeficácia experiências, aprendizagem vicariante, persuasão verbal-social e estados físicos e emocionais (Bandura, 1997; Usher \& Pajares, 2008, 2009). Estas fontes de autoeficácia atuam em conjunto e influenciam-se reciprocamente ao longo do desenvolvimento (Usher \& Pajares, 2009). A informação proveniente destas fontes é seleccionada e incorporada nos julgamentos pessoais de capacidade, em função da idade e de fatores académicos, sociais e culturais (Ahn et al., 2016; Chen \& Usher, 2013; Flores et al., 2017; Fouad \& Santana, 2017). Neste âmbito, as atividades escolares desempenham um papel primordial, pois proporcionam cognições e sentimentos sobre experiências de sucesso e fracasso, oportunidades de modelagem e de feedback (Lent, 2004). Este é um contexto de contínua aprendizagem, em que as crianças vão criando e recriando as suas próprias representações académicas e sociais, acompanhadas de sentimentos e emoções que sedimentam a conceção sobre si próprias como aprendentes e como pessoas futuramente inseridas em contextos sociais e de trabalho. As crianças tendem a atribuir significado às aprendizagens numa perspetiva de futuro (e.g. bom futuro, arranjar trabalho, tirar a carta de condução) (Teixeira, 2014), evidenciando alicerces precoces do planeamento da carreira na relação motivacional entre conteúdos académicos e aplicação na vida e na carreira (Dedmond, Schwallie-Giddis, \& Strong, 2012).

A literatura tem também mostrado o papel determinante da autoeficácia nos domínios vocacionais e académicos (e.g. Brown \& Lent, 2017; Bandura et. al., 2001; Lent \& Brown, 2017;
Lopes \& Teixeira, 2012; Teixeira, 2008a), sendo que a falta de modelos e incentivos são obstáculos à construção da carreira (Heppner, 2009; Herlihy \& Watson, 2012; Prime, Bernstein, Wilkins, \& Bekk, 2013). Em crianças, a persuasão do professor, sob forma de feedback, determina a perceção de competência, os interesses, a motivação dos alunos por áreas curriculares, nomeadamente matemática (Laranjeira \& Teixeira, 2012). As crenças de autoeficácia cumprem um papel crucial na carreira (Pajares, 2002), especialmente na formação de projetos não tradicionais de género, na carreira das mulheres (Betz, 1994). Contudo, apesar das evidências do efeito negativo dos estereótipos no desenvolvimento da carreira das mulheres nas áreas da ciência, tecnologia e matemática, a medida dos seus efeitos tem sido negligenciada (Deemer, Lin, Graham, \& Soto, 2016).

\section{Avaliação dos Interesses}

De um ponto de vista descritivo, a avaliação dos interesses na infância pode ser vista como prematura. A estabilidade dos interesses vocacionais emerge após a consolidação da identidade, através do confronto com a crise psicossocial identidade versus confusão de identidade, descrita por Erikson (1968) para a adolescência. Contudo, apesar do consenso que existe relativamente à origem da formação dos interesses, pouco se sabe sobre o seu estabelecimento e evolução na infância, visto que a maioria dos estudos são conduzidos com adolescentes e adultos (Swanson, 1999; Tracey, 2002). Deste modo, a avaliação dos interesses durante a infância tem como significado compreender a formação do autoconceito e consequentemente identificar variáveis envolvidas nesse processo. Os dados da avaliação dos interesses chamam a atenção para a associação entre interesses e diferenciação cognitiva, associando a formação dos interesses à capacidade da criança diferenciar competência em diferentes atividades (Lent, 2004). Nos estudos realizados com interesses, esta premissa teórica é apoiada pela presença de um fator geral, que congrega muitos dos itens e uma parte significativa da variância, nos procedimentos de análise fatorial exploratória (Tracey \& Ward, 1998; Tracey, 2002). No entanto, com alguma frequência, este 
fator geral dos interesses também aparece em amostras de adolescentes, particularmente quando os itens têm características ipsativas (Teixeira, 2008b).

$\mathrm{Na}$ avaliação dos interesses, o modelo de Holland (1997) é um dos mais usados na investigação e intervenção (e.g. Almeida, Ahmetoglu, \& Chamorro-Premuzic, 2014; Leuty, Hansen \& Speaks, 2016; Morgan, Bruin, \& Bruin, 2015; Sung, Cheng, \& Wu, 2016). Este modelo organiza os interesses de acordo com a taxonomia RIASEC: R (Realista), I (Investigativo) A (Artístico), S (Social), E (Empreendedor) e C (Convencional), a que corresponde a mesma organização das atividades profissionais, académicas ou sociais. Numa caracterização geral dos tipos pelas atividades, no Realista predominam tarefas práticas, estruturadas e concretas, no Investigativo atividades criativas e de investigação, no Artístico atividades criativas, no Social contacto interpessoal e relação de ajuda, no Empreendedor contacto interpessoal em contextos organizacionais ou materiais e no Convencional tarefas com dados explícitos, ordenados e sistemáticos (Holland, 1997). O ponto essencial desta teoria reside na premissa de que as pessoas estão motivadas para procurarem ambientes que melhor combinem com a constelação dos seus interesses e que o ajustamento entre pessoa e ambiente resulta em satisfação (Leuty et al., 2016).

Este modelo é representado numa estrutura hexagonal (Holland, 1997) em que cada ângulo corresponde a um tipo de personalidade. Segundo os princípios calculus e consistência os tipos são ordenados pela proximidade-contiguidade psicológica, em que as correlações entre tipos adjacentes (R-I, I-A, A-S, S-E, E-C, C-R) são mais elevadas do que entre tipos a dois passos de distância, (R-I > R-A), que, por sua vez, são superiores às obtidas entre os tipos opostos (R-A > R-S) (Barros, 2015; Holland, 1997; Teixeira, Barros, \& Janeiro, 2010; Tracey \& Caulum, 2015). Os ângulos opostos, como Realista e Social, representam diferentes atividades que implicam características psicológicas diferentes, que, neste caso, traduzem uma atração para lidar com coisas vs. pessoas, de acordo com a classificação de Prediger (1999). Num estudo com adolescentes portugueses, as correlações entre tipos adjacentes - Realista e Investigativo (0.38) ou Empreendedor e Convencional (0.56) revelam magnitude superior às dos tipos opostos Realista e Social (0.02) ou Convencional e Artístico (0.13), sendo estes dados similares aos obtidos em amostras do Brasil (Teixeira et al., 2010). O princípio da diferenciação decorre da consistência e pressupõe que num perfil individual se destacam 2-3 tipos, sendo responsável pelas diferenças intra e interindividuais dos tipos de personalidade (Holland, 1997).

\section{Objetivos do Estudo}

Este estudo examina as qualidades psicométricas do Inventário de Atividades para Crianças - Revisto (ICA-R) (Tracey \& Ward, 1998), de acordo com os quadros teóricos de Gottfredson (1996), Holland (1997) e Lent e colaboradores (1994). No plano de validação do ICA-R ao contexto português, são objetivos analisar: (1) grau de precisão da medida, (2) estrutura dos interesses e da perceção de competências das crianças, (3) efeito de género e das preferências escolares nos resultados, (4) natureza intercultural do ICA-R e do modelo de Holland e (5) implicações dos resultados para a investigação e educação de carreira no $1^{\circ}$ ciclo.

\section{Método}

\section{Participantes}

A amostra é de conveniência, inclui 228 crianças (56\% do sexo feminino), com idades entre 9 e 11 anos (mediana=9), que frequentavam escolas públicas $(35 \%)$ e privadas $(65 \%)$ de Lisboa, durante o ano letivo de 2014/2015. Os participantes eram alunos do $4^{\circ}$ ano de escolaridade, assegurando-se a compreensão do conteúdo dos itens neste nível de escolaridade.

\section{Instrumento}

\section{Inventário de Atividades para Crianças}

Com o objetivo de analisar os interesses e as perceções de competência das crianças, Tracey e Ward (1998) desenvolveram o Inventário de Atividades para Crianças - Revisto (ICA-R), destinado a avaliar a relação entre estas duas dimensões do autoconceito. Este instrumento baseou-se na tipologia das personalidades de Holland (1997), sendo as atividades adaptadas ao 
contexto infantil. A relevância do ICA-R prendese com os raros instrumentos que avaliam na infância os interesses e a autoeficácia; em Portugal, as medidas que avaliam estes construtos (e.g. Barros, 2015; Teixeira, 2008ab) destinam-se prioritariamente aos adolescentes, jovens adultos e adultos.

O ICA-R encontra-se dividido em duas partes, cada uma constituída por 30 itens. A primeira parte corresponde à medida dos interesses e contém uma lista de atividades do dia-a-dia das crianças (e.g. "Inventar uma história"), cuja resposta deve indicar o quanto gostam de as realizar, numa escala Likert de 5 pontos (1- Não gosto nada; 5 - Gosto muito). A segunda parte do questionário apresenta a mesma lista de atividades, mas é pedido às crianças que indiquem a perceção de competência, com utilização da escala de 5 pontos (1 - Sou muito mau/má; 5 Sou muito bom/boa). Os itens estão organizados em seis subescalas, com correspondência aos seis tipos de personalidade propostos por Holland (1997). Cada subescala é composta por 5 itens (e.g. Realista - "Consertar um brinquedo"; Investigativo - "Ver coisas ao microscópio"; Artístico - "Brincar com barro ou plasticina"; Social - "Ensinar alguém a ler"; Empreendedor "Vender coisas aos outros"; Convencional "Fazer tabelas ou gráficos". Para além dos 30 itens originais, o autor introduziu de forma experimental outros itens, entre os quais dois sobre o papel do género nos interesses e nas perceções de competência (e.g. "Coisas que os rapazes gostam de fazer") (Tracey, 2002).

Nos estudos originais, a análise fatorial indica uma estrutura dos interesses e das perceções de competência das crianças associada aos fatores género e contexto das atividades, progredindo com a idade o ajustamento das dimensões ao modelo hexagonal (Tracey \& Ward, 1998; Tracey, 2002). Nestes estudos americanos, os alfas de Cronbach variam entre $.61 \mathrm{e} .81$ nas subescalas de competência, e entre .60 e .80 nas subescalas de interesse (Tracey \& Ward, 1998). Tracey (2002) atribui a moderada homogeneidade de algumas das subescalas ao número reduzido de itens por subescala e ao grau incipiente da consistência e diferenciação dos interesses na infância. Para além do ICA-R, as crianças responderam também a um questionário de dados sociodemográficos, que continha a questão das preferências curriculares.

\section{Procedimento}

Para utilização do instrumento foi pedida permissão ao autor principal, sendo os conteúdos traduzidos por dois investigadores em separado, e as duas traduções comparadas com uma outra versão previamente usada em contexto português (David, Paixão, \& Silva, 2009). Ao longo do processo de adaptação, o conteúdo de alguns itens foi modificado no sentido de lhes conferir maior proximidade ao mundo infantil - por exemplo no item "Observar alguém a arranjar uma televisão", a palavra "televisão" foi substituída por "computador", tornando o conteúdo mais atual. Também se considerou adequado adicionar exemplos a alguns itens, antecipando possíveis dificuldades de interpretação - ao item "Compreender como as coisas funcionam" foi adicionado "Exemplo: O ciclo da água". Adicionalmente, o formato de alguns itens da escala de competência foi alterado para transformar as atividades em tarefas ativas (e.g. ao item "Ouvir música" foi adicionada a componente de atenção - "Ouvir música com atenção").

O projeto de investigação foi aprovado pela Comissão de Deontologia da Faculdade de Psicologia da Universidade de Lisboa e aceite o pedido de aplicação dos questionários pelas direções de seis escolas da área metropolitana de Lisboa, que acederam participar no estudo. Em colaboração com os professores titulares das turmas, enviou-se o consentimento informado aos encarregados de educação, que incluía informações sobre a participação das crianças no estudo, objetivos da pesquisa, condições de confidencialidade dos dados e caráter voluntário da participação, e ainda o contacto do investigador, caso fosse necessário clarificar algum assunto. Os questionários foram administrados em sala de aula no grupo-turma, sendo previamente explicado aos alunos a natureza do estudo e condições de liberdade e confidencialidade da sua participação.

Foi efetuado um estudo piloto para averiguar a compreensão dos itens e o tempo necessário para a resposta, que se estimou em cerca de 10 minutos. Os resultados foram analisados com recurso ao software SPSS, versão 22.0. 


\section{Resultados}

\section{Preferências Expressas pelas Disciplinas} Escolares, por Género. Nas preferências pelas principais disciplinas do $1^{\circ}$ ciclo, $48 \%$ das crianças escolheu Estudo do Meio, 30\% Matemática e $22 \%$ Português. Os dados de uma tabela de contingência mostram associação significativa entre as variáveis sexo e disciplina preferida $\left[\chi_{(2)}^{2}=17.00 ; p<.001\right]$. Existem mais rapazes do que raparigas a preferirem matemática e mais raparigas do que rapazes a escolherem língua portuguesa. Nas preferências por estudo do meio, os grupos possuem relativa equivalência.

\section{Inventário de Atividades para Crianças}

Distribuição dos Itens. Em geral, as respostas aos itens apresentam uma distribuição próxima à normal, à exceção dos itens 4 ("Conversar com amigos"), 9 ("Ouvir música") e 20 (“Observar coisas ao microscópio”), em que existe pouca variabilidade de resposta.

Análise Fatorial Exploratória. Nos resultados dos interesses e das perceções de competência procedeu-se à análise em componentes principais, numa solução imposta de seis fatores, de acordo com o scree plot e a definição teórica das escalas. As soluções foram rodadas por método varimax.

$\mathrm{Na}$ escala de interesses, a solução explica cerca de $42 \%$ da variância e o valor de KMO (0.71) indica uma validade aceitável da análise. O Quadro 1 apresenta uma síntese da matriz rodada, em que se incluem os pesos fatoriais superiores a |0.40|, considerando-se três itens com um peso superior a $|0.32|$.

O Fator I explica cerca de $16 \%$ da variância e congrega quatro dos cinco itens da subescala Social, dois da Convencional, dois da Artística e ainda o item das atividades ditas femininas. É de salientar que este fator não possui características de um fator geral, quer pela proporção da variância explicada, quer pelo conteúdo dos itens, que corresponde às atividades tradicionalmente femininas nas áreas sociais, artísticas e convencionais. O Fator II é responsável por $10 \%$ da variância, sendo definido por três dos cinco itens convencionais e dois realistas. De acordo com a estrutura hexagonal, estes dois tipos são adjacentes. O Fator III explica $6 \%$ da variância e agrega três dos cinco itens da subescala Realista, um da Investigativa, um da Empreendedora e ainda o item das atividades masculinas. O Fator IV é definido quatro dos cinco itens da subescala Investigativa, e explica $6 \%$ da variância. O Fator $\mathrm{V}$ explica cerca de $5 \%$ da variância, agrega três dos cinco itens da subescala Artística e ainda as saturações elevadas e inversas dos dois itens de género- saturação positiva $(0.42)$ do item feminino e negativa (-0.43) do item masculino. $\mathrm{O}$ fator VI explica $4 \%$ da variância e é definido por três dos cinco itens da subescala Empreendedor e um item Social, sendo estes dois tipos contíguos na estrutura hexagonal.

O mesmo procedimento foi conduzido para a escala de competências percebidas, sendo imposta também uma solução de seis fatores, responsável por $47 \%$ da variância total. O valor KMO (0.72) indica uma razoável correlação entre os itens e uma validade de análise aceitável. O Quadro 2 apresenta uma síntese dos dados da matriz rodada, considerando os pesos fatoriais superiores a $|0.40|$. Sete itens foram incluídos com pesos superiores a |0.31|.

O Fator I explica 16\% da variância e agrega três dos cinco itens da subescala Social, dois da Convencional, um da Artística, o item das atividades femininas com saturação positiva (0.42) e o das atividades masculinas com saturação negativa (-0.34). Este fator tem semelhanças com o primeiro da escala de Interesses, quer na percentagem de variância explicada, quer no conteúdo dos itens. O Fator II explica $10 \%$ da variância e é definido por quatro dos cinco itens Artísticos e dois Sociais, observando-se a saturação de 0.51 do item feminino. O Fator III explica $6 \%$ da variância, agrega cinco itens Investigativos, dois da subescala Realista, e o item 19 (Realista) possui também saturação neste fator (0.35), embora com peso fatorial superior no fator V. O Fator IV explica 5\% da variância e é definido por três itens da subescala Convencional, por dois itens da subescala Realista e um item Empreendedor. O Fator V explica 5\% da variância e inclui três itens Empreendedores, um Realista e o item das atividades masculinas (0.60). É de referir que o item 21 da subescala Artística, com alusão a uma tarefa manipulativa, tem saturação (0.42) neste 
Quadro 1. Síntese da Matriz Fatorial Rodada por Varimax (Escala de Interesses)

\begin{tabular}{|c|c|c|c|c|c|c|c|c|}
\hline \multicolumn{9}{|c|}{ Fatores } \\
\hline \multicolumn{3}{|c|}{1} & \multicolumn{3}{|c|}{2} & \multicolumn{3}{|c|}{3} \\
\hline Tipo & Item & Peso & Tipo & Item & Peso & Tipo & Item & Peso \\
\hline S & $\begin{array}{l}\text { Ensinar alguém a } \\
\text { ler }\end{array}$ & .68 & $\mathrm{C}$ & Somar números & .66 & $\mathrm{R}$ & Martelar pregos & .66 \\
\hline$S$ & $\begin{array}{l}\text { Ajudar os outros a } \\
\text { sentirem-se bem }\end{array}$ & .65 & $\mathrm{R}$ & $\begin{array}{l}\text { Observar uma } \\
\text { construção }\end{array}$ & .62 & Gen & $\begin{array}{l}\text { Fazer coisas que os } \\
\text { rapazes gostam }^{\text {a }}\end{array}$ & .56 \\
\hline $\mathrm{C}$ & $\begin{array}{l}\text { Manter as coisas } \\
\text { arrumadas }\end{array}$ & .59 & $\mathrm{R}$ & $\begin{array}{l}\text { Observar alguém a } \\
\text { reparar um } \\
\text { computador }\end{array}$ & .56 & I & Desmontar coisas & .54 \\
\hline $\mathrm{C}$ & $\begin{array}{l}\text { Organizar um } \\
\text { roupeiro }\end{array}$ & .55 & $\mathrm{C}$ & $\begin{array}{l}\text { Contar e ordenar } \\
\text { coisas }\end{array}$ & .52 & $\mathrm{R}$ & $\begin{array}{l}\text { Consertar um } \\
\text { brinquedo }\end{array}$ & .52 \\
\hline S & $\begin{array}{l}\text { Tomar conta de } \\
\text { crianças mais } \\
\text { novas }\end{array}$ & .53 & $\mathrm{C}$ & $\begin{array}{l}\text { Fazer tabelas ou } \\
\text { gráficos }\end{array}$ & .48 & $\mathrm{E}$ & $\begin{array}{l}\text { Organizar jogos } \\
\text { para os outros } \\
\text { jogarem }\end{array}$ & .48 \\
\hline S & $\begin{array}{l}\text { Ajudar pessoas } \\
\text { doentes }\end{array}$ & .52 & & & & $\mathrm{R}$ & $\begin{array}{l}\text { Construir coisas } \\
\text { (e.g. puzzles) }\end{array}$ & .46 \\
\hline Gen & $\begin{array}{l}\text { Fazer coisas que as } \\
\text { raparigas gostam }^{\text {a }}\end{array}$ & .50 & & & & & & \\
\hline A & $\begin{array}{l}\text { Cantar, } \\
\text { acompanhando a } \\
\text { música }\end{array}$ & .48 & & & & & & \\
\hline A & Ouvir música & .38 & & & & & & \\
\hline \multicolumn{9}{|c|}{ Fatores } \\
\hline & 4 & & & 5 & & & 6 & \\
\hline Tipo & Item & Peso & Tipo & Item & Peso & Tipo & Item & Peso \\
\hline I & $\begin{array}{l}\text { Observar coisas ao } \\
\text { microscópio }\end{array}$ & .63 & A & $\begin{array}{l}\text { Inventar uma } \\
\text { história }\end{array}$ & .71 & $\mathrm{E}$ & $\begin{array}{l}\text { Dizer aos outros o } \\
\text { que devem fazer }\end{array}$ & .72 \\
\hline I & $\begin{array}{l}\text { Misturar coisas } \\
\text { para ver o que } \\
\text { acontece }\end{array}$ & .62 & A & Desenhar & .69 & $\mathrm{E}$ & $\begin{array}{l}\text { Ser o líder/chefe } \\
\text { do grupo }\end{array}$ & .61 \\
\hline I & $\begin{array}{l}\text { Compreender } \\
\text { como as coisas } \\
\text { funcionam }\end{array}$ & .60 & Gen & $\begin{array}{l}\text { Fazer coisas que os } \\
\text { rapazes gostam }\end{array}$ & -.43 & $\mathrm{E}$ & $\begin{array}{l}\text { Vender coisas aos } \\
\text { outros }\end{array}$ & .58 \\
\hline \multirow[t]{2}{*}{ I } & $\begin{array}{l}\text { Assistir a um } \\
\text { documentário } \\
\text { científico }\end{array}$ & .52 & Gen & $\begin{array}{l}\text { Fazer coisas que as } \\
\text { raparigas gostam }{ }^{\text {a }}\end{array}$ & .42 & $S$ & $\begin{array}{l}\text { Conversar com os } \\
\text { amigos }\end{array}$ & .38 \\
\hline & & & A & $\begin{array}{l}\text { Brincar com barro } \\
\text { ou plasticina }\end{array}$ & .32 & & & \\
\hline
\end{tabular}

Nota: R - Realista, I - Investigativo, A - Artístico, S - Social, E - Empreendedor, C - Convencional, Gen - Género. ${ }^{a}$ Item que satura em mais do que um fator.

fator. Por fim, o Fator VI explica 5\% da variância e é definido por dois itens do tipo Empreendedor.

Precisão. Os alfas de Cronbach variam entre $.49 \mathrm{e}$ .63 na escala de interesses e entre .50 e .66 na escala de competências percebidas (Quadro 3). Alguns destes indicadores situam-se abaixo do limite considerado aceitável para a homogeneidade das medidas (<.60) (Murphy \& Davidshofer, 2001) e são ligeiramente inferiores aos do estudo original (Tracey \& Ward, 1998) mas próximos dos obtidos com as crianças mais novas, no contexto Português (David, Paixão, \& Silva, 2009, 2015).

Distribuição dos Resultados nas Subescalas. O Quadro 3 apresenta indicadores descritivos das subescalas: mínimo (Min), máximo (Max), média (M), desvio-padrão (DP), assimetria (Sk) e curtose $(\mathrm{Ku})$. Relativamente às hierarquias dos resultados, as médias são superiores nas subescalas do tipo Artístico e inferiores nas do tipo Realista, sendo estes dados similares aos obtidos por David e colaboradores $(2009,2015)$. Os índices de 
Quadro 2. Síntese da Matriz Rodada por Varimax (Escala de Competências)

\begin{tabular}{|c|c|c|c|c|c|c|c|c|}
\hline \multicolumn{9}{|c|}{ Fatores } \\
\hline \multicolumn{3}{|c|}{1} & \multicolumn{3}{|c|}{2} & \multicolumn{3}{|c|}{3} \\
\hline Tipo & Item & Peso & Tipo & Item & Peso & Tipo & Item & Peso \\
\hline $\mathrm{C}$ & $\begin{array}{l}\text { Organizar um } \\
\text { roupeiro }\end{array}$ & .72 & A & $\begin{array}{l}\text { Ouvir música com } \\
\text { atenção }\end{array}$ & .68 & I & $\begin{array}{l}\text { Observar coisas ao } \\
\text { microscópio }\end{array}$ & .68 \\
\hline $\mathrm{C}$ & $\begin{array}{c}\text { Manter as coisas } \\
\text { arrumadas }\end{array}$ & .67 & $\mathrm{~A}$ & $\begin{array}{l}\text { Cantar, } \\
\text { acompanhando a } \\
\text { música }\end{array}$ & .61 & I & $\begin{array}{l}\text { Assistir com } \\
\text { atenção a um } \\
\text { documentário } \\
\text { científico }\end{array}$ & .67 \\
\hline$S$ & $\begin{array}{l}\text { Tomar conta de } \\
\text { crianças mais } \\
\text { novas }\end{array}$ & .57 & $S$ & $\begin{array}{c}\text { Conversar com os } \\
\text { amigos }\end{array}$ & .60 & I & $\begin{array}{l}\text { Misturar coisas } \\
\text { para ver o que } \\
\text { acontece }\end{array}$ & .57 \\
\hline$S$ & $\begin{array}{c}\text { Ensinar alguém a } \\
\text { ler }\end{array}$ & .56 & Gen & $\begin{array}{l}\text { Fazer coisas que as } \\
\text { raparigas gostam }^{\text {a }}\end{array}$ & .51 & I & $\begin{array}{l}\text { Compreender } \\
\text { como as coisas } \\
\text { funcionam }\end{array}$ & .53 \\
\hline$S$ & $\begin{array}{l}\text { Ajudar pessoas } \\
\text { doentes }\end{array}$ & .54 & $S$ & $\begin{array}{l}\text { Ajudar os outros a } \\
\text { sentirem-se bem }\end{array}$ & .45 & $\mathrm{R}$ & Martelar pregos & .51 \\
\hline A & Desenhar & .44 & A & $\begin{array}{c}\text { Brincar com barro } \\
\text { ou plasticina }^{\mathrm{a}}\end{array}$ & .32 & $\mathrm{R}$ & $\begin{array}{c}\text { Observar } \\
\text { atentamente } \\
\text { alguém a reparar } \\
\text { um computador }\end{array}$ & .43 \\
\hline Gen & $\begin{array}{l}\text { Fazer coisas que as } \\
\text { raparigas gostam a }\end{array}$ & .42 & A & $\begin{array}{l}\text { Inventar uma } \\
\text { história }\end{array}$ & .31 & I & Desmontar coisas & .38 \\
\hline Gen & $\begin{array}{l}\text { Fazer coisas que os } \\
\text { rapazes gostam }\end{array}$ & -.34 & & & & $\mathrm{R}$ & $\begin{array}{l}\text { Consertar um } \\
\text { brinquedo }^{\text {a }}\end{array}$ & .35 \\
\hline \multicolumn{9}{|c|}{ Fatores } \\
\hline & 4 & & & 5 & & & 6 & \\
\hline Tipo & Item & Peso & Tipo & Item & Peso & Tipo & Item & Peso \\
\hline $\mathrm{C}$ & Somar números & .70 & Gen & $\begin{array}{l}\text { Fazer coisas que os } \\
\text { rapazes gostam }^{\text {a }}\end{array}$ & .60 & $\mathrm{E}$ & $\begin{array}{c}\text { Ser o líder/chefe } \\
\text { do grupo }\end{array}$ & .82 \\
\hline $\mathrm{C}$ & $\begin{array}{l}\text { Fazer tabelas ou } \\
\text { gráficos }\end{array}$ & .66 & $\mathrm{R}$ & $\begin{array}{l}\text { Consertar um } \\
\text { brinquedo }^{\text {a }}\end{array}$ & .47 & $\mathrm{E}$ & $\begin{array}{l}\text { Dizer aos outros o } \\
\text { que devem fazer }\end{array}$ & .82 \\
\hline $\mathrm{R}$ & $\begin{array}{l}\text { Observar } \\
\text { atentamente uma } \\
\text { construção }\end{array}$ & .52 & $\mathrm{E}$ & $\begin{array}{l}\text { Tentar convencer } \\
\text { os teus pais a } \\
\text { fazerem algo que } \\
\text { queres }\end{array}$ & .44 & & & \\
\hline $\mathrm{R}$ & $\begin{array}{c}\text { Construir coisas } \\
\text { (e.g. puzzles) }\end{array}$ & .47 & $\mathrm{E}$ & $\begin{array}{l}\text { Vender coisas aos } \\
\text { outros }\end{array}$ & .43 & & & \\
\hline $\mathrm{E}$ & $\begin{array}{l}\text { Organizar jogos } \\
\text { para os outros } \\
\text { jogarem }^{a}\end{array}$ & .42 & A & $\begin{array}{c}\text { Brincar com barro } \\
\text { ou plasticina }^{a}\end{array}$ & .42 & & & \\
\hline $\mathrm{C}$ & $\begin{array}{c}\text { Contar e ordenar } \\
\text { coisas }\end{array}$ & .38 & $\mathrm{E}$ & $\begin{array}{l}\text { Organizar jogos } \\
\text { para os outros } \\
\text { jogarem }^{\text {a }}\end{array}$ & .38 & & & \\
\hline
\end{tabular}

Nota: R - Realista, I - Investigativo, A - Artístico, S - Social, E - Empreendedor, C - Convencional, Gen - Género. ${ }^{a}$ Item que satura em mais do que um fator.

assimetria e achatamento indicam respostas com distribuição próxima à normal em todas as subescalas, confirmando a sensibilidade do instrumento às diferenças individuais.

Correlações. As correlações entre interesses e perceções de competência do mesmo tipo variam entre $.61 \mathrm{e} .75$, e observam-se coeficientes superiores a .30 entre tipos de interesses e algumas escalas adjacentes de competências percebidas (e.g. R-I; A-S).

Diferenças entre Grupos

Género. Na escala de interesses (Quadro 5), os resultados médios são significativamente 
Quadro 3. Estatísticas Descritivas das Subescalas

\begin{tabular}{|c|c|c|c|c|c|c|c|c|c|c|c|c|c|c|}
\hline \multirow{2}{*}{ Escala } & \multicolumn{10}{|c|}{ Interesses } & \multicolumn{4}{|c|}{ Perceções de Competência } \\
\hline & $\alpha$ & Min & Max & M & DP & Sk & $\mathrm{Ku}$ & $\alpha$ & Min & $\operatorname{Max}$ & M & DP & $\mathrm{Sk}$ & $\mathrm{Ku}$ \\
\hline $\mathrm{R}$ & .63 & 7 & 25 & 16.53 & 3.94 & -.02 & -.37 & .66 & 5 & 25 & 16.59 & 4.07 & -.05 & -.25 \\
\hline I & .50 & 8 & 25 & 20.45 & 3.21 & -.95 & .07 & .56 & 9 & 25 & 20.18 & 3.30 & -.72 & .20 \\
\hline A & .49 & 11 & 25 & 21.33 & 2.82 & -.96 & .74 & .50 & 11 & 25 & 20.68 & 2.93 & -.82 & .61 \\
\hline$S$ & .58 & 13 & 25 & 21.15 & 2.83 & -.63 & -.16 & .63 & 9 & 25 & 19.97 & 3.17 & -.49 & .11 \\
\hline E & .49 & 8 & 25 & 18.12 & 3.53 & -.53 & .20 & .53 & 7 & 25 & 18.10 & 3.61 & -.47 & -.02 \\
\hline $\mathrm{C}$ & .63 & 6 & 25 & 17.92 & 3.75 & -.48 & -.20 & .63 & 8 & 25 & 18.27 & 3.67 & -.50 & .10 \\
\hline
\end{tabular}

Quadro 4. Matriz de Correlações entre Interesses e Competências Percebidas

\begin{tabular}{|c|c|c|c|c|c|c|}
\hline & \multicolumn{6}{|c|}{ Perceções de Competência } \\
\hline Interesses & $\mathrm{R}$ & $\mathrm{I}$ & A & $\mathrm{S}$ & $\mathrm{E}$ & $\mathrm{C}$ \\
\hline $\mathrm{R}$ & $.71 * *$ & $.40 * *$ & .01 & $.14^{*}$ & $.23 * *$ & $.26 * *$ \\
\hline I & $.34 * *$ & $.69 * *$ & .03 & .10 & .00 & $.18^{* *}$ \\
\hline A & .02 & .11 & $.73 * *$ & $.34 * *$ & .05 & .25 \\
\hline S & $.14^{*}$ & $.19^{*}$ & $.40 * *$ & $.70 * *$ & .12 & $.37 * *$ \\
\hline $\mathrm{E}$ & $.24 * *$ & $.18^{*}$ & .12 & $.14^{*}$ & $.61 * *$ & $.14 *$ \\
\hline $\mathrm{C}$ & $.25^{*}$ & $.23 *$ & $.16 *$ & $.32 * *$ & .08 & $.75 * *$ \\
\hline
\end{tabular}

Nota: $* * p<.001 ; * p<.05$.

A negrito correlações iguais ou superiores a .30 .

superiores para os rapazes nas subescalas do tipo Realista e Empreendedor, sendo superiores para as raparigas nas subescalas do tipo Artístico e Social. A dimensão do efeito é média e baixa nas diferenças da subescala do tipo Empreendedor. $\mathrm{Na}$ escala de competências percebidas (Quadro 5), as diferenças mantêm o mesmo padrão. É ainda observada uma diferença significativa e favorável às raparigas na subescala do tipo Convencional. A maioria dos efeitos é de dimensão média, e nos tipos Empreendedor e Convencional é reduzida.
Preferência pelas Disciplinas. Nos resultados da ANOVA a um fator, o efeito da preferência pelas três principais disciplinas do $1^{\circ}$ ciclo de ensino é significativo nas subescalas de interesse do tipo Artístico $\quad\left(\mathrm{F}_{(2 ; 189)}=7.19 ; \quad p=.001 ; \quad \eta_{\mathrm{p}}^{2}=0.071\right) \quad \mathrm{e}$ Social $\left(\mathrm{F}_{(2 ; 192)}=3.22 ; \quad p=.042 ; \quad \eta_{\mathrm{p}}^{2}=0.033\right)$, nas subescalas de competência Realista $\left(\mathrm{F}_{(2 ; 192)}=6.42\right.$; $\left.p=.002 ; \eta_{\mathrm{p}}^{2}=0.063\right)$ e Artística $\left(\mathrm{F}_{(2 ; 191)}=6.23\right.$; $p=.002 ; \eta_{\mathrm{p}}^{2}=0.062$ ), e a dimensão do efeito é baixa em todos os casos.

Quadro 5. Diferenças de Género Entre as Médias das Subescalas do Inventário de Atividades para Crianças

\begin{tabular}{lccccccc}
\hline & \multicolumn{7}{c}{ Género } \\
\hline \multirow{2}{*}{ Subescalas } & Média & DP & Média & DP & t & $p$ & $\eta 2 p$ \\
\cline { 2 - 7 } Interesse R & 18.33 & 3.74 & 15.12 & 3.51 & 6.48 & $* *$ & 0.16 \\
Interesse I & 20.48 & 3.35 & 20.41 & 3.12 & 0.14 & 0.89 & - \\
Interesse A & 20.06 & 3.00 & 22.31 & 2.24 & -6.11 & $* *$ & 0.16 \\
Interesse S & 20.21 & 2.90 & 21.90 & 2.55 & -4.61 & $* *$ & 0.09 \\
Interesse E & 18.89 & 2.70 & 17.50 & 3.98 & 3.03 & $*$ & 0.04 \\
Interesse C & 17.44 & 4.19 & 18.28 & 3.34 & -1.59 & 0.11 & - \\
Competência R & 18.39 & 3.85 & 15.17 & 3.68 & 6.34 & $* *$ & 0.16 \\
Competência I & 20.38 & 3.29 & 20.02 & 3.33 & 0.79 & 0.431 & - \\
Competência A & 19.54 & 3.27 & 21.58 & 2.27 & -5.24 & $* *$ & 0.12 \\
Competência S & 18.98 & 3.27 & 20.73 & 2.87 & -4.23 & $* *$ & 0.08 \\
Competência E & 18.95 & 3.26 & 17.43 & 3.75 & 3.18 & $*$ & 0.04 \\
Competência C & 17.68 & 3.71 & 18.74 & 3.58 & -2.10 & $*$ & 0.02 \\
\hline
\end{tabular}

Nota: $* * p<.001 ; * p<.05$.

$\mathrm{R}=$ Realista, $\mathrm{I}=$ Investigativo, $\mathrm{A}=$ Artístico, $\mathrm{S}=$ Social, $\mathrm{E}=$ Empreendedor, $\mathrm{C}=$ Convencional 
$\mathrm{Na}$ análise de comparação múltipla à posteriori (teste LSD), as crianças com preferência nas disciplinas de português $(M=22.45, D P=2.24, p<.001)$ ou estudo do meio $(M=21.55, D P=2.68, p<.05)$ apresentam médias superiores nos interesses do tipo Artístico, comparativamente ao grupo da preferência a matemática $(M=20.39, \quad D P=3.01)$, e as que preferem português $(M=21.77, D P=2.24, p<.05)$ ou estudo do meio $(M=21.41, D P=2.84, p<.05)$ demonstram ainda interesses sociais mais elevados, em comparação com colegas que gostam de matemática $(M=20.44, D P=3.07)$.

No que diz respeito às perceções de competência, observa-se uma diferença significativa nas médias do tipo Realista favorável, às crianças que preferem matemática $(M=17.84$, $D P=3.73, \quad p<.001)$, comparativamente às que gostam de português $(M=14.90, D P=4.28)$, e as que preferem português $(M=21.43, D P=2.52$, $p<.05)$ ou estudo do meio $(M=20.97, D P=2.79$, $p<.05)$ perceberem-se como mais competentes no domínio artístico, comparativamente aos colegas com preferência matemática $(M=19.59, D P=3.12)$.

\section{Discussão}

No âmbito da literatura sobre a formação dos interesses e competências percebidas (e.g. Gottfredson, 1996, 2002; Brown \& Lent, 2017; Lent \& Brown, 2017, Lent et al., 1994) e da organização dos interesses pela tipologia RIASEC (Holland, 1997), este estudo examina as qualidades psicométricas do Inventário de Atividades para Crianças - Revisto (ICA-R) (Tracey \& Ward, 1998). A discussão centra-se nos indicadores da consistência interna da medida e das componentes da validade de construto, nomeadamente (1) estrutura interna, (2) associação entre perceções de competência e interesses, (3) relação entre estas duas dimensões e variáveis critério género e preferências escolares e (4) equivalência dos resultados com os das amostras americanas.

\section{Precisão}

Os coeficientes alfa de Cronbach colocam reservas à interpretação dos resultados, de acordo com Murphy e Davidshofer (2001), situando-se entre .49 e .63 na escala de interesses e entre .50 e
.66 na escala de competências percebidas. Estes coeficientes são ligeiramente inferiores aos do estudo original (Tracey \& Ward, 1998), mas convergentes com os obtidos por David, Paixão e Silva (2015) em crianças mais novas. Estes dados corroboram a associação entre homogeneidade da medida e idade-ano de escolaridade, no sentido de que a estabilidade e consistência dos interesses emerge no próprio desenvolvimento cognitivo (Seligman, 1994). De acordo com Tracey (2002), o número reduzido de itens por subescala pode também concorrer para baixos índices de precisão.

\section{Validade}

Estrutura Interna. Os dados dos interesses e das competências percebidas tendem a apresentar uma estrutura similar, que confirmam a equivalência teórica entre competências percebidas e interesses nas crianças, tal como é preconizado pela teoria de Holland (1997).

Nas duas estruturas de análise exploratória dos dados, a percentagem de variância explicada pelo primeiro fator e respetivos conteúdos não sugerem a presença de um fator geral, ao contrário do que acontece no estudo original (Tracey \& Ward, 1998). Em contrapartida, nos dados desta amostra, nas duas soluções o Fator I reúne itens de tipo social, convencional e o item do género feminino, corroborando o efeito de género nos interesses (Gottfredson, 2002). O conteúdo dos itens que definem os seis fatores dos interesses está associado às caraterísticas dos tipos de personalidade (Fatores II, II e IV) e ao fator género (Fatores I, III e V). Os fatores das competências percebidas seguem a mesma tendência, com ordem diferente dos fatores. Apesar de não existir um perfeito ajustamento à estrutura hexagonal, observa-se a tendência de cada fator reunir os itens de um mesmo tipo de personalidade e associar itens das subescalas adjacentes, sugerindo, por um lado organização dos interesses e perceção de competências pelo modelo de Holland (1997), e, por outro lado, os dados indiciam cristalização do autoconceito infantil por áreas mais extensas de atividades circunscritas a dois-três tipos (e.g., E-R-I; C-S-A), associando o fator género (e.g., M-E-R-I; F-C-SA) ${ }^{1}$, como confirmado por Gottfredson (2002).

M- item masculino associado a itens dos tipos Empreendedor, Realista, Investigativo (Fator III) e F- item feminino associado a itens dos tipos Convencional, Social e Artístico (Fator I). 
Nas duas soluções em componentes principais há indicadores de convergência com a definição teórica das escalas (Holland, 1997) e com características de desenvolvimento das crianças (Gottfredson, 2002), embora o estudo não siga metodologia longitudinal. Estes dados contêm ainda indicadores de diferenciação, consistência e de continuidade psicológica (calculus) entre os tipos adjacentes, que corroboram o modelo de Holland (1997).

\section{Associação entre Perceções de Competência e}

Interesses. Os dados também demonstram correlações elevadas entre interesses e perceções de competência dentro de um mesmo tipo e correlações altas entre tipos contíguos (e.g. R-I; E-C). Apesar das correlações não permitirem identificar a direção da relação, estes dados são corroborados pelas evidências do efeito direto das competências percebidas nos interesses do mesmo tipo (Laranjeira \& Teixeira, no prelo) e sugerem confirmação do modelo sociocognitivo da formação dos interesses (Lent et al., 1994). As correlações entre resultados dos tipos de interesses com os das competências percebidas adjacentes (e.g. R-I; A-S) confirmam a validade de construto, no sentido da consistência dentro de cada tipo e da diferenciação entre os tipos (Holland, 1997).

\section{Variáveis Critério}

Disciplinas Preferidas. Os dados sugerem também o efeito das preferências escolares no autoconceito vocacional. As crianças que gostam de português tendem a sentir-se mais competentes e a preferir atividades dos tipos Artísticos e Social, enquanto as que preferem matemática privilegiam competências e interesses do tipo Realista. Estes dados corroboram ainda os princípios da consistência e diferenciação (Holland, 1997), no sentido que um mesmo tipo reúne interesses, competências e preferências escolares, e os diferentes tipos transparecem diferenças nos conteúdos.

Género. Os dados relativos ao efeito da variável sexo nos resultados dos interesses e competências são observados nas estruturas fatoriais, nos resultados do $t$ student e nas disciplinas preferidas.

Nas estruturas fatoriais dos interesses, o item que representa as atividades do sexo feminino está associado aos conteúdos do tipo Social,
Convencional e Artístico (Fatores I e V), enquanto o item do sexo masculino às atividades do tipo Realista, Investigativo e Empreendedor (Fator III), e estes dados tendem a ser replicados nos resultados das competências percebidas. Nos resultados médios, as raparigas confirmam superioridade das médias nos tipos Artísticos e Sociais e os rapazes nos tipos Realista e Empreendedor, e no mesmo sentido, os rapazes preferem mais a matemática do que as raparigas. A superioridade dos rapazes nos interesses e competências com base comum na matemática é corroborada pelos indicadores de que o autoconceito dos rapazes é mais elevado a matemática (Unlu \& Ertekin, 2013) mas também pelas evidências do maior reforço social dado aos rapazes a matemática (Gunderson et al., 2012) e das expetativas mais elevadas dos professores sobre as capacidades matemáticas dos rapazes (Lazarides \& Watt, 2015). A suposta implicação da aprendizagem na relação entre autoconceito a matemática e género é corroborada pelas evidências dos rapazes percecionam mais feedback positivo do professor a matemática do que as raparigas (Laranjeira \& Teixeira, no prelo). No caso específico da matemática, as raparigas quando percecionam feedback positivo a matemática tendem a revelar mais interesses ligados ao cálculo, constituindo ainda o professor um fator moderador entre género e interesses artísticos para os rapazes que percebem feedback positivo a língua portuguesa (Laranjeira \& Teixeira, no prelo).

$\mathrm{O}$ efeito do género nos interesses e competências percebidas é corroborado internacionalmente e ao longo de gerações (e.g. Iskander et al., 2013; Teixeira, 2014; Tyler, 1965) em crianças (e.g. David et al., 2015; Laranjeira \& Teixeira, 2012), adolescentes (e.g. Baptista \& Teixeira, 2012; Teixeira et al., 2010) e jovens adultos (e.g. Teixeira, 2010). Em todos estes estudos há convergência quanto à orientação motivacional das raparigas no domínio da relação pessoal e das atividades artísticas e do envolvimento dos rapazes nas áreas da atividade física, dados, máquinas e ferramentas, tal como corroborado em diferentes amostras e culturas (e.g. Guntern, Korpershoek, \& Werf, 2016; Iskander et al., 2013; Prime et al., 2015). O presente estudo tem a mais-valia de analisar 
interesses e competências percebidas na infância e corrobora o género como determinante na formação do autoconceito infantil (Bussey \& Bandura, 1999; Gottfredson, 1981; 1996), apesar de se salientarem outros indicadores de natureza académica, familiar e social na formação do autoconceito (Laranjeira \& Teixeira, 2012; RubieDavies, 2006; Teixeira \& Ferreira, no prelo). Inevitavelmente, estes resultados confirmam a inegável perpetuação das diferenças de género ao longo de gerações, e conduzem à reflexão sobre o contributo das intervenções no âmbito da psicologia educacional, no sentido de esbater este efeito. É necessário analisar estes temas com neutralidade ideológica e repensar estratégias nas intervenções para crianças e educadores.

Validade Intercultural. Apesar das limitações, os resultados confirmam as qualidades psicométricas do ICA-R e a validade intercultural da medida e do modelo de Holland (1997) para investigação e intervenção. As crianças portuguesas e americanas tendem a representar atividades, interesses e competências de acordo com critérios equivalentes, e as diferenças observadas podem ser atribuídas à idade e aos contextos específicos das crianças, sendo a amostra americana mais heterogénea em níveis de escolaridade (Tracey \& Ward, 1998). Uma vez mais se confirmam as potencialidades do modelo de Holland (1997) em diferentes culturas, sendo estes dados corroborados por estudos anteriores (e.g., Barros, 2015; Morgan et al., 2015; Teixeira et al., 2010).

\section{Limitações}

Este estudo tem limitações relativas à dimensão e natureza da amostra, nomeadamente incluir apenas crianças do $4^{\circ}$ ano e da região de Lisboa. Em relação às medidas, o ICA-R evidencia coeficientes de homogeneidade relativamente baixos e usa os mesmos conteúdos para avaliar interesses e competências, o que pode introduzir redundância nos resultados da segunda parte. A metodologia transversal concorre para algumas limitações, visto que o estudo foca dimensões em desenvolvimento.

\section{Conclusões}

O conjunto dos dados sugere que as crianças desde muito cedo revelam um autoconceito relativamente organizado, com consistência e diferenciação dos interesses e competências percebidas, em que sobressaem influências de género e do ambiente escolar. As implicações destes dados fazem-se sentir ao nível da investigação que procura compreender os fatores de desenvolvimento vocacional das crianças, que envolve, entre outros, componentes sociais e escolares, no âmbito dos processos de aprendizagem e motivação. Nas implicações para a prática, os resultados deste estudo podem servir de referência para organizar atividades educativas, que considerem os fatores que determinam a cristalização prematura da imagem social da criança e, consequentemente limitam as opções disponíveis tanto na aprendizagem como nas escolhas de carreira. Estas intervenções devem constituir oportunidades das crianças desenvolverem capacidades, de formarem representações pessoais livres de constrangimentos de natureza social e cultural e conceberem oportunidades sem limitações sociais. Perspetiva-se a intervenção com crianças alargada aos educadores, nomeadamente pais e professores, no sentido de criarem oportunidades e quadros de referência facilitadores de um desenvolvimento pleno e harmonioso da criança.

Os dados evidenciam as potencialidades do ICA-R na investigação sobre a formação do autoconceito, nomeadamente acerca dos mecanismos psicológicos responsáveis pelo efeito dos estereótipos no desenvolvimento da carreira. Nesse sentido, perspetivam-se estudos para aprofundar o processo da formação das crenças de autoeficácia e dos interesses, que utilizem fontes de autoeficácia relacionadas com a aprendizagem e desenvolvimento da carreira (e.g., desempenhos, modelos, persuasão e suporte emocional). A possibilidade de analisar longitudinalmente estas dimensões e variáveis pode esclarecer sobre os determinantes do autoconceito académico e vocacional e identidade vocacional ao longo da vida. Poderá ainda ser pertinente adaptar a versão mais recente do ICA (Tracey \& Caulum, 2015), construído especificamente para esbater as diferenças de género nos resultados. 


\section{Referências}

Ahn, H. S., Usher, E. L., Butz, A., \& Song, M. (2016). Cultural differences in the understanding of modelling and feedback as sources of self-efficacy information. British Journal of Educational Psychology, 86(1), 112-136. doi:10.1111/bjep.12093

Almeida, P. I. L., Ahmetoglu, G., \& ChamorroPremuzic, T. (2014). Who wants to be an Entrepreneur? The relationship between vocational interests and individual differences in entrepreneurship. Journal of Career Assessment, 22(1) 102-112. doi: $10.1177 / 1069072713492923$

Bandura, A. (1977). Self-Efficacy: Toward a unifying theory of behavioral change. Psychological Review, 84, 191-215.

Bandura, A. (1997). Self-efficacy: The exercise of control. New York: W.H. Freeman and Company.

Bandura, A., Barbaranelli, C., Caprara, G. V., \& Pastorelli, C. (2001). Self-efficacy beliefs as shapers of children's aspirations and career trajectories. Child Development Journal, 72(1), 187-206.

Baptista, J. A. C., \& Teixeira, M. O. (2012). OPUS: uma nova medida de interesses para o aconselhamento da carreira. Revista Psicologia Militar, 21, 41-55.

Barros, A. F. (2015). Contributos para a validação do Questionário de Busca Autodirigida (Versão Brasileira do SDS - Self-Directed Search) em estudantes Cabo-Verdianos. Revista Iberoamericana de Diagnóstico y Evaluación - e Avaliação Psicológica, 40(2), 62-72.

Betz, N. E. (1994). Basic issues and concepts in career counseling for women. In W. Bruce Walsh \& S. H. Osipow (Eds.), Career Counseling for women. (pp. 1-42). New Jersey: Lawrence Erlbaum Associates.

Brown, S. D., \& Lent, R. (2017). Social Cognitive Career Theory in a diverse world: Closing thoughts. Journal of Career Assessment, 25(1) 173-180. doi: 10.1177/10690727166.

Bussey, K., \& Bandura, A. (1999). Social cognitive theory of gender development and differentiation. Psychological Review, 106(4), 676-713.
Chen, J. A., \& Usher, E. L. (2013). Profiles of the sources of science self-efficacy. Learning \& Individual Differences, 24, 11-21. doi: 10.1016/j.lindif.2012.11.002.

David, R., Paixão, M. P., \& Silva, J. T. (2009). Interesses e competências percebidas em crianças do ensino básico. Psychologica, 51, 73-92.

David, R., Paixão, M. P., \& Silva, J. T. (2015). Interesses e competências percebidas na infância: Um estudo com crianças do ensino básico. Revista Brasileira de Orientação Profissional, 16(1), 49-58.

Dedmond, R. M., Schwallie-Giddis, P., \& Strong, S. E. (2012). Kindergarten through eigth grade. In D. Capuzzi, \& M. Stauffer, Career Counseling: Fundations, Perspectives and Applications (pp. 315-340). New York: Routledge.

Deemer, E. D., Lin, C., Graham, R., \& Soto, C. (2016). Development and validation of a measure of threatening gender stereotypes in science: A factor mixture analysis. Journal of Career Assessment, 24(1), 145-161. doi: 10.1177/1069072714565772.

Erikson, E. H. (1968). Identity youth and crisis. New York: W. W. Norton \& Company.

Flores, L. Y., Navarro, R. L., \& Rasheed, S. (2017). The state of SCCT research in relation to social class: Future directions. Journal of Career Assessment, 25(1), 6-23. doi: 10.1177/1069072716658649.

Fouad, N., \& Santana, M. C. (2017). SCCT and Underrepresented Populations in STEM Fields: Moving the Needle. Journal of Career Assessment, 25(1) 24-39. doi: $10.1177 / 1069072716658324$.

Gottfredson, L. S. (1981). Circumscription and compromise: A developmental theory of occupational aspirations [Monograph]. Journal of Counseling Psychology, 28(6), 545-579.

Gottfredson, L. S. (1996). Gottfredson's theory of circumscription and compromise. In D. Brown, \& L. Brooks, (Eds.), Career choice and development ( $3^{\mathrm{a}}$ ed., pp. 179-232). San Francisco: Jossey-Bass.

Gottfredson, L. S. (2002). Gottfredson's theory of circumscription, compromise, and self creation. In D. Brown (Ed.), Career Choice 
and Development $\left(4^{\text {th }}\right.$ ed., pp. 85-148). San Francisco: Jossey-Bass.

Gunderson, E. A., Ramirez, G., Levine, S. C., \& Beilock, S. I. (2012). The role of parents and teachers in the development of gender-related math attitudes. Sex Roles, 66, 156-166. doi:10.1007/s11199-011-9996-2.

Guntern, S., Korpershoek, H., \& van der Werf, G. (2016). Prestige added to Holland's vocational interest scales for the prediction of medical students' aspired work environments. Journal of Career Assessment, 24(2), 333-346. doi: $10.1177 / 1069072715580418$

Hendricks, K. S. (2016). The Sources of selfefficacy: Educational research and implications for music. National Association for Music Education, 35(1) 32-38. doi:10.1177/8755123315576535.

Heppner, P. (2009). Empowering men's life choices: An examination of gender and sexual orientation. In N. Gysbers, M. Heppner \& J. Johnston, Career Counseling: Contexts, processes, and techniques (pp. 101-123). Alexandria: American Counseling Association.

Herlihy, B., \& Watson, Z. (2012). Gender issues in career counseling. In: D. Capuzzi, \& M. Stauffer, Career Counseling: Fundations, Perspectives and Applications (pp. 497 - 523). New York: Routledge.

Herr, E., \& Cramer, S. (1996). Career guidance and counseling through the life span: systematic approaches. (5th ed.). New York: Longman.

Høigaard, R., Kovač, V. B., Øverby, N. C., \& Haugen, T. (2015). Academic self-efficacy mediates the effects of school psychological climate on academic achievement. School Psychology Quarterly, 30(1), 64-74. doi:10.1037/spq0000056

Holland, J. L. (1997). Making vocational choices: A theory of vocational personalities and work environments ( $3^{\mathrm{a}}$. ed.). Odessa, FL: Psychological Assessment Resources.

Iskander, E. T., Gore, P. A. Jr., Furse, C., \& Bergenson (2013). Gender differences in expressed interests in engineering-related fields ACT 30-year data analysis identified trends and suggested avenues to reverse trends. Journal of Career Assessment, 21(4) 599-613. doi:10.1177/1069072712475290

Laranjeira, M. (2015). A formação do autoconceito vocacional em contexto académico e social (Dissertação de mestrado não publicada). Faculdade de Psicologia da Universidade de Lisboa, Portugal.

Laranjeira, M., \& Teixeira, M. O. (no prelo). Versão portuguesa da Escala de Feedback do Professor: Estudo preliminar com crianças do $4^{\circ}$ Ano. Paidéia.

Lazarides, R., \& Watt, H. G. (2015). Girls' and boys' perceived mathematics teacher beliefs, classroom learning environments and mathematical career intentions. Contemporary Educational Psychology, 41, 51-61. doi:10.1016/j.cedpsych.2014.11.005

Lent, R. W. (2004). Social cognitive career theory, career education, and school-to work transition: Building a theoretical framework for career preparation. In M. C. Taveira (Coord.), Desenvolvimento vocacional ao longo da vida: Fundamentos, princípios $e$ orientações (pp. 13-23). Coimbra: Almedina.

Lent, R. W., Brown, S. D., \& Hackett, G. (1994). Toward a unifying social cognitive theory of career and academic interest, choice, and performance [Monograph]. Journal of Vocational Behavior, 45, 79-122.

Lent, R. W., \& Brown, S. D. (2017). Social cognitive career theory in a diverse world: Guest editors' introduction. Journal of Career Assessment, 3(1), 3-5. doi: 10.1177/10690727 166.

Leuty, M. E., Hansen, J. C., \& Speaks, S. Z. (2016). Vocational and leisure interests: A profile-level approach to examining interests. Journal of Career Assessment, 24(2) 215-239. doi: 10.1177/1069072715580321.

Lopes, A. R., \& Teixeira, M. O. (2012). Projetos de carreira, autoeficácia e sucesso escolar em ambiente multicultural. Revista Brasileira de Orientação Profissional, 13(1), 7-14.

Morgan, B., Bruin, G. P., \& Bruin, K. (2015). Constructing Holland's hexagon in South Africa: Development and initial validation of the South African Career Interest Inventory. Journal of Career Assessment, 3(3) 493511.doi: 10.1177/1069072714547615. 
Murphy, K. R., \& Davidshofer, C. O. (2001). Psychological testing: Principles and applications $\left(5^{\text {th }}\right.$ ed.). Prentice Hall. New Jersey.

Pajares, F. (2002). Gender and perceived selfefficacy in self-regulated learning. Theory Into Practice, 41(2), 116-125. doi: 10.1207/s15430421tip4102_8.

Pinxten, M., Marsh, H. W., De Fraine, B., Van Den Noortgate, W., \& Van Damme, J. (2014). Enjoying mathematics or feeling competent in mathematics? Reciprocal effects on mathematics achievement and perceived math effort expenditure. British Journal of Educational Psychology, 84(1), 152-174. doi: 10.1111/bjep.1202.

Prediger, D. J. (1999). Basic structure of workrelevant abilities. Journal of

Counseling Psychology, 46, 173-184.

Prime, D. R., Bernstein, B. L., Wilkins, K. G., \& Bekk, J. M. (2015). Measuring the advising alliance for female graduate students in science and engineering: An emerging structure. Journal of Career Assessment, 23(1) 64-78. doi: 10.1177/106907271452308 6

Rubie-Davies, C. M. (2006). Teacher expectations and student self-perceptions: Exploring relationships. Psychology in the Schools, 43(5), 537-552. doi:10.1002/pits.20169.

Seligman, L. (1994). Developmental career counseling and assessment ( $2^{\mathrm{a}}$ ed.). Thousand Oaks, CA: Sage Publications.

Silva, R. (2016). Estilos parentais e formação do autoconceito vocacional. Um estudo com crianças do primeiro ciclo (Dissertação de mestrado não publicada). Faculdade de Psicologia da Universidade de Lisboa, Portugal.

Sung, Y. T., Cheng, Y. W., \& Wu, J. S. (2016). Constructing a situation-based career interest assessment for junior high school students and examining their interest structure. Journal of Career Assessment, 24(2) 347-365. doi: 10.1177/1069072715580419

Super, D. E. (1990). A life-span, life-space approach to career development. In D. Brown \& L. Brooks (Eds.), Career choice and development: Applying contemporary theories to practice (2nd ed., pp. 197-261). San Francisco: Jossey-Bass.

Swanson, J. L. (1999). Stability and change in vocational interests. In M. L. Savickas \& R. L. Spokane (Eds.), Vocational interests meaning, measurement, and counseling use (pp.135-158). Palo Alto, CA: DaviesBlack.

Teixeira, M. O. (2008a). A Escala Multidimensional de Auto-eficácia Percebida: Um estudo exploratório numa amostra de estudantes do ensino superior. Revista Iberoamericana de Diagnóstico y Evaluación - e Avaliação Psicológica, 25(1), 141-157.

Teixeira, M. O. (2008b). Inventário de Interesses Vocacionais de Jackson (JVIS). In L. Almeida, M. Simões \& M. Gonçalves (Eds.). Avaliação Psicológica. Instrumentos validados para a população portuguesa, II ( $2^{\mathrm{a}}$ Ed. Revista) (57-74). Coimbra: Quarteto.

Teixeira, M. O. (2009). O desenvolvimento dos projectos profissionais em contexto escolar: As questões da idade e do género. Psicologia e Educação, VII(1), 127-134.

Teixeira, M. O. (2010). Indicadores de validade do Inventário de interesses JVIS num grupo profissional. In C. Nogueira, I. Silva, L. Lima, A. T. Almeida, R. Cabecinhas, R. Gomes, C. Machado, A. Maia, A. Sampaio \& M. C. Taveira (Eds.), Actas do VII Simpósio Nacional de Investigação em Psicologia (pp. 191-197). Retirado de http://www.actassnip20 10.com.

Teixeira, M. O. (2014). Infância: Tempo de crescer, sonhar e aprender. Comunicação no Congresso de Psicólogos nas Escolas - Coimbra 2014. Coimbra: Faculdade de Psicologia e Ciências da Educação da Universidade de Lisboa.

Teixeira, M. O., Barros, A., \& Janeiro, J. (2010). Análise transcultural dos interesses. Resultados do SDS em Amostras de Portugal e do Brasil. In C. Nogueira, I. Silva, L. Lima, A. T. Almeida, R. Cabecinhas, R. Gomes, C. Machado, A. Maia, A. Sampaio \& M. C. Taveira (Eds.). Actas do VII Simpósio Nacional de Investigação em Psicologia (pp. 3796-3805). Retirado de http://www.actassni p2010.com. 
Teixeira, M. O., \& Ferreira, I. A. (no prelo). Fatores familiares e escolares nas aspirações, na autoeficácia e no sucesso académico: um estudo exploratório com estudantes de $9^{\circ}$ ano. Revista Portuguesa de Psicologia.

Tracey, T. G. (2002). Development of interests and competency beliefs: A 1 year longitudinal study of fifth- to eighth-grade students using the ICA-R and structural equation modeling. Journal of Counseling Psychology, 49(2), 148-163. doi:10.1037/0022-0167.49.2.148.

Tracey, T. J. G., \& Caulum, D. (2015). Minimizing gender differences in children's interest assessment: Development of the Inventory of Children's Activities-3 (ICA-3). Journal of Vocational Behavior, 87, 154-160.

Tracey, T. G., \& Ward, C. C. (1998). The structure of children's interests and competence perceptions. Journal of Counseling Psychology, 45(3), 290-303.

Tyler, L. E. (1965). The psychology of human differences ( $3^{\mathrm{a}}$ ed.). New York: Prentice-Hall.

Unlu, M., \& Ertekin, E. (2013). The relationship between mathematics teaching self-efficacy and mathematics self-efficacy. Procedia Social and Behavioral Sciences, 106, 30413045. Doi: 10.1016.

Usher, E. L., \& Pajares, F. (2008). Sources of selfefficacy in school: Critical review of the literature and future directions. Review of Educational Research, 78(4), 751-796. doi:10.3102/0034654308321456.

Usher, E. L., \& Pajares, F. (2009). Sources of selfefficacy in mathematics: A validation study. Contemporary Educational Psychology, 34(1), 89-101. doi:10.1016/j.cedpsych.2008.09.002

Vaz Serra, A. (1988). O auto-conceito. Análise Psicológica, 6(2), 101-110. 